\title{
THE VALUE OF DIGITAL DERMATOSEOPY IN THE DIAGNOSIS AND TREATMENT OF PRECANGEROUS SKIN LESIONS
}

\section{Podac Claudiu', Bordeianu Ion², Poalelungi Tudorel', Iordache I.V.', Bosnac Nida', Abdulazis T.', Panculescu F.G.'}

${ }^{1}$ Emergency County Hospital "Sf. Apostol Andrei” Constanța

${ }^{2}$ Faculty of Medicine, University "Ovidius" of Constanța

${ }^{3}$ P.h M.D. Dermatology and Venereology, Constanța

${ }^{4}$ P.h M.D. Plastic surgery, Constanţa

\begin{abstract}
We propose that through the presented study, to strengthen the value of the pre-excision digital dermatoscopy by emphasizing its accuracy compared to the histopathological examination in the diagnosis and treatment of precancerous lesions of the skin for which the histopathological examination of certainty can only be performed after post-excision.
\end{abstract}

Keywords: dermatoscopy, precancerous skin lesion, histopatological.

\section{Introduction}

As a non-invazive mainly used tehnique, digital dermatoscopy had considerably improved the accuracy of skin lesion diagnosis; this procedure enables the in vivo observation of the skin with the visualization of the morphological structures in the dermis and papilary dermis, which are not routinely discernible to the unaided eye.Dermatoscopy is a helpful diagnostic tool for the clinical examination of non-melanoma skin cancer and had a notable impact on early diagnosis of melanoma $(1,2)$.

Torule outorconfirmagiven dermatoscopic diagnosis, it involves the recognition of specific structures, or their absence; this task can be accomplished using a 'bottom-up' or 'top-down' ' strategy (3).

Essentially, dermatoscopy is a new procedure of gross, histopathological examination of the skin performed by clinicians, since the structures and colors observed by dermatoscopy have specific correlations on histopathological examination, clinicians must learn the definition and histopathological correlation of dermatoscopic structures and colors (4).

The digital dermatoscope can see the entire width of the lesion horizontally, the structure and the colors observed are generally limited in depth to the papillary dermis and do not allow 
the assessment of the lesion at a cellular level, however dermatoscopy has the capability to sequentially monitor the lesions, which in turn they can provide information about the lesion biology and the growth dynamics $(5,6)$.

\section{History}

The surface microscopy of the skin began in 1663 when Kolhaus investigated the vessels of the nail matrix with a microscope $(7,8)$. The application of immersion oil in light microscopy was described in 1878 by Abbe, and his principle was transferred by the german dermatologist Unna in 1893 to skin surface microscopy.

Johann Saphier published in 1920 a series of communications using a new diagnostic tool similar to a binocular microscope with a built-in light source and introduced for the first time the term "dermatoscopy" $(7,9)$.

Skin surface microscopy was further developed in the United States by Goldman in the 1950 which published a series of articles on new devices on what he called "Dermoscopy" $(7,10)$.

The advantage of surface microscopy was clearly established for the first time in 1971, by Rona MacKie for improving the differential diagnosis of benign from malignant lesions and the preoperative diagnosis of pigmented skin lesions $(7,11)$.

In 1989 in Hamburg was held the first Conference on Skin Microscopy, and in 2001 in Rome took place the Netmeeting of Dermatoscopy which was the first international meeting of this kind $(7,12,13)$.

Dermatoscopy is today a routine technique, and the digital video dermatoscopy is successfully used in teledermoscopy, through which we can send images from a cabinet to specialized centers (second opinion).

\section{Material and method}

In order to confirm the clinical value of digital dermatoscopy in the diagnosis and treatment of precancerous lesions of the skin, we conducted a prospective study on a group of patients with precancerous skin tumors (where the diagnosis was based on clinical signs) to which we checked the concordances between the diagnosis of digital dermatoscopy and histopathological diagnosis.

The study was spread over a period of 3 years, respectively 01.01.2014-31.12.2016, being represented in cases of patients diagnosed in ambulatory with precancerous conditions and which have benefited of digital dermatoscopy , one day surgical treatment, histopathological examination in paraffin and a small part of them of immunohistochemical examination.

For the establishmentof the study group, we have set criteria for inclusion / exclusion from the start in order to be able to obtain scientifically and technically conclusive results.

It should be noted that inclusion in the group was not restrictive, accepting patients of different sexes and ages with various associated diseases, and exclusion criteria did not include noncompliant patients (who did not show post-operative checks in accordance with the indications).

Postoperative controls were performed every 3 months clinically and biannually from dermatoscopic point of view for at least 1 year after surgery.

\section{Resulits}

The group of patients included a total of 29 cases, of both sexes, aged between 20-85 years, from rural and urban areas, with primary, secondary and / or higher education studies.

Also, the classification of the lesions of the patients included in the study regarding the type of precancerous lesions is diverse such as actinic keratosis, lentigo maligna, Recklinghausen neurofibromatosis, Bowen disease.

Surgical excision was performed according to current international standards at a distance of 2-3 $\mathrm{mm}$ in the surface in healthy tissue and deeply into fat and / or fascial planes. Closure of the post-excision defect was performed by direct suturing and / or free skin grafting (splitthickness or full- thickness).

The statistical survey of the group showed the following: 


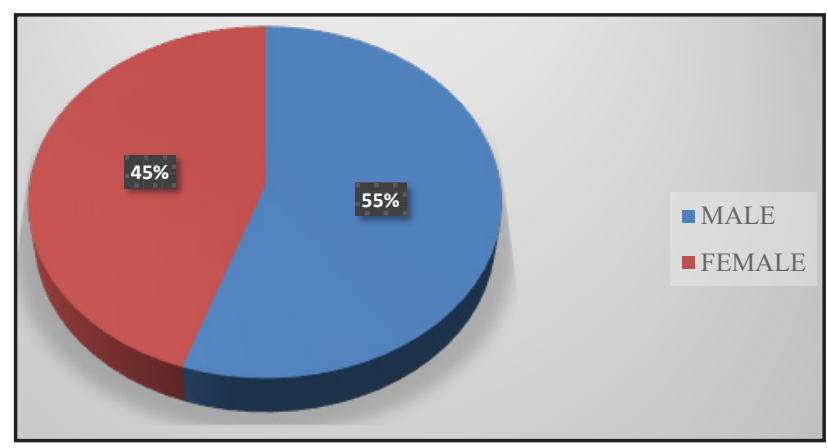

Figure 1. Gender distribution of patients with precancerous lesions

The sex distribution of patients with precancerous lesions included in our study reveals a majority of $55 \%$ for male gender and $45 \%$ for female gender.

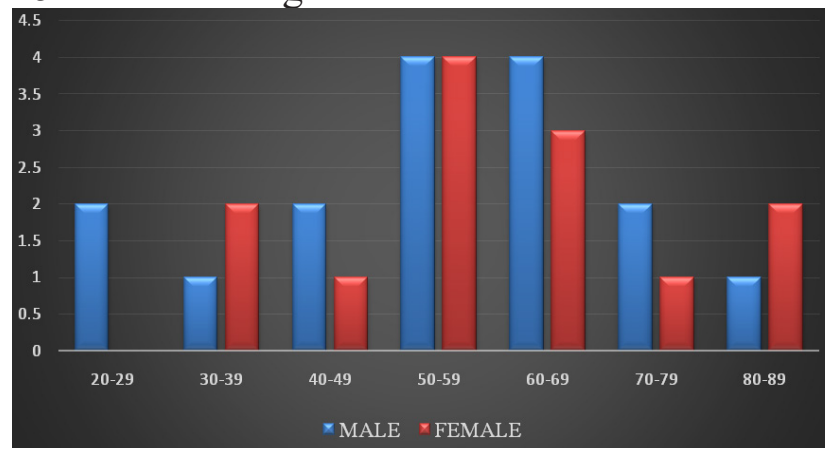

Figure 2. The repartition of patients with precancerous lesions over decades of age

The distribution of cases with precancerous skin lesions included in the study over decades of age revealed a higher incidence of cases in the 6 th decade to both sexes, followed by the 7 th decade with the mention that in the 4th and 9th decade the majority of cases were female cases, and for the 5th, 7th and 8th decade there were predominant cases of males.

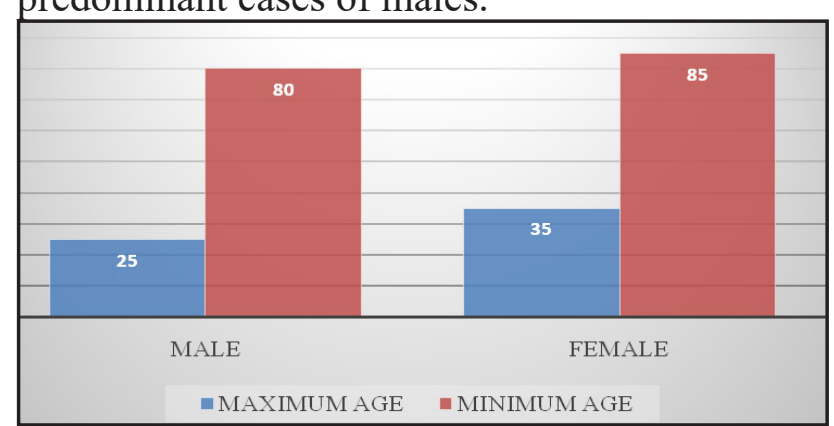

Figure 3. Distribution of patients with precancerous lesions by age group depending on gender

Presentation to a specialist and therapeutic indication is relatively similar in both sexes by age group.

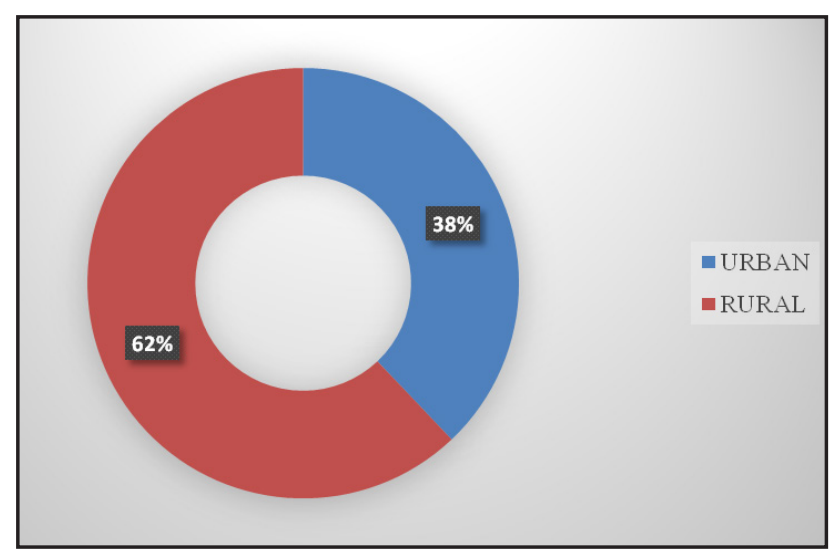

Figure 4. Distribution of patients with precancerous lesions according to their home environment

It can be seen a net predominance of those from the rural area, representing $62 \%$ of the total studied cases.

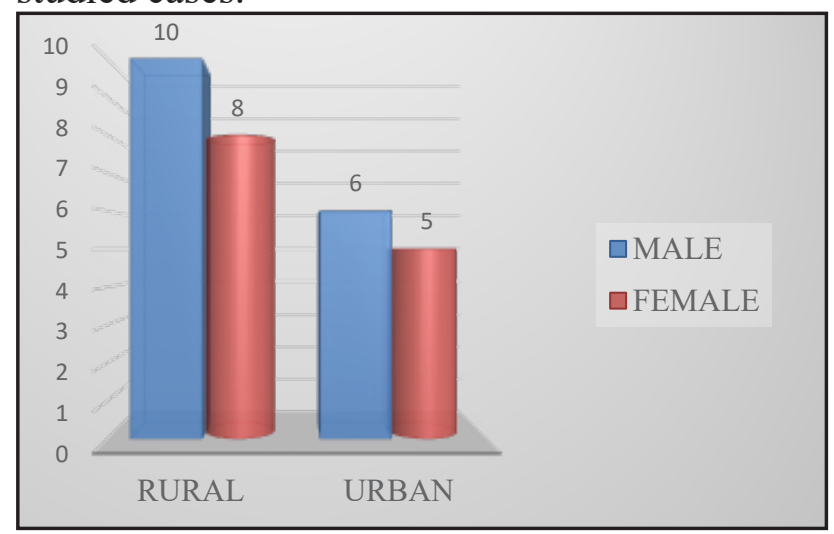

Figure 5. Distribution of patients with precancerous lesions by area of origin and gender

The patients with precancerous lesions included in the study, according to area of origin related on the gender, shows that men are more affected in both rural and urban area.

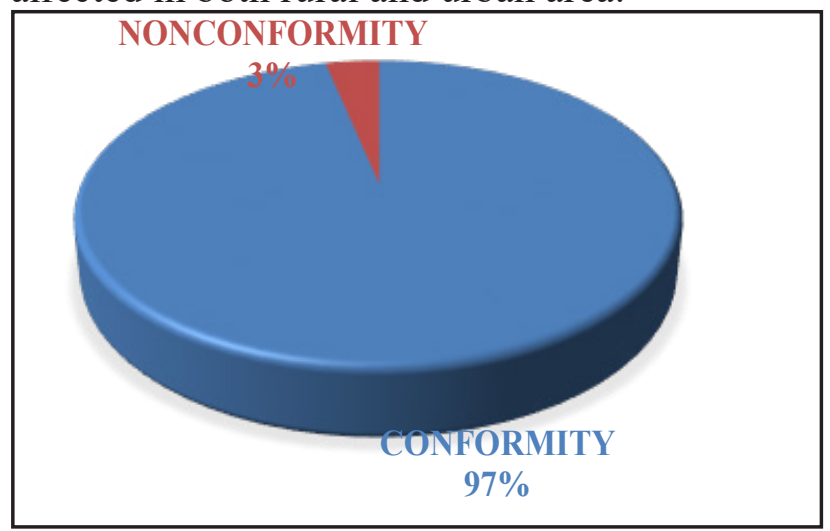

Figure 6. Conformity / Nonconformity - Dermatoscopic Exam $\rightarrow$ Histopathological Exam

We note that the overlap between dermatoscopic and histopathological results was 
97\%; The result confirms the value of digital dermatoscopy proper diagnosis of precancerous skin lesions.

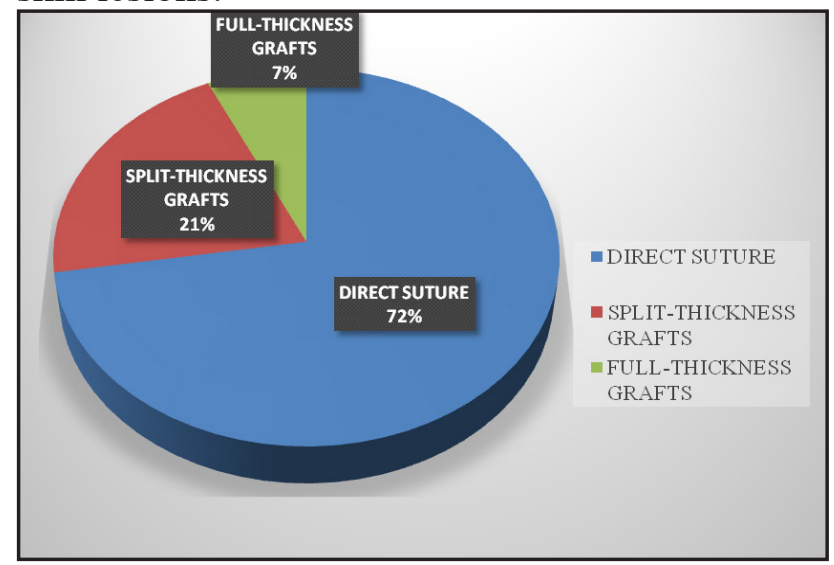

Figure 7. Distribution methods of closing the postexcision defect

Regarding the method of closing the postexcision defect, the most used was the direct suturing technique with $72 \%$, followed by splitthickness grafts with $21 \%$ respectively, by fullthickness grafts with $7 \%$.

Illustrative clinical cases.

CASE I.

Before surgery-19-09-2015

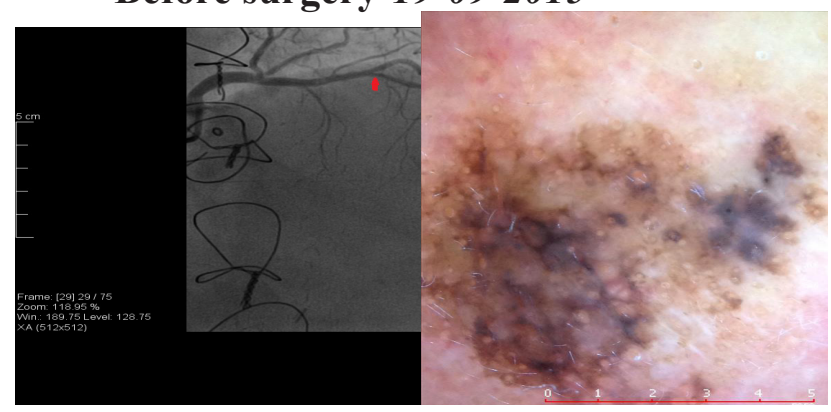

Clinical appearance Dermatoscopic appearance

Dermatoscopic diagnosis: Lentigo maligna (white-blue wave, asymmetric follicular pigmentation, marginal rhomboid structures.

Differential diagnosis with:

1. Superficial malignant melanoma on lentigo maligna;

2. Solar Lentigo.

Recommendations: Surgical excision with side edges $0.5 \mathrm{~cm}$

The result of the histopathological examination was in conformity with the examination of digital dermatoscopy (superficial melanoma on lentigo maligna).

The post-excision defect was covered by auto-transplantation of full-thickness grafts.
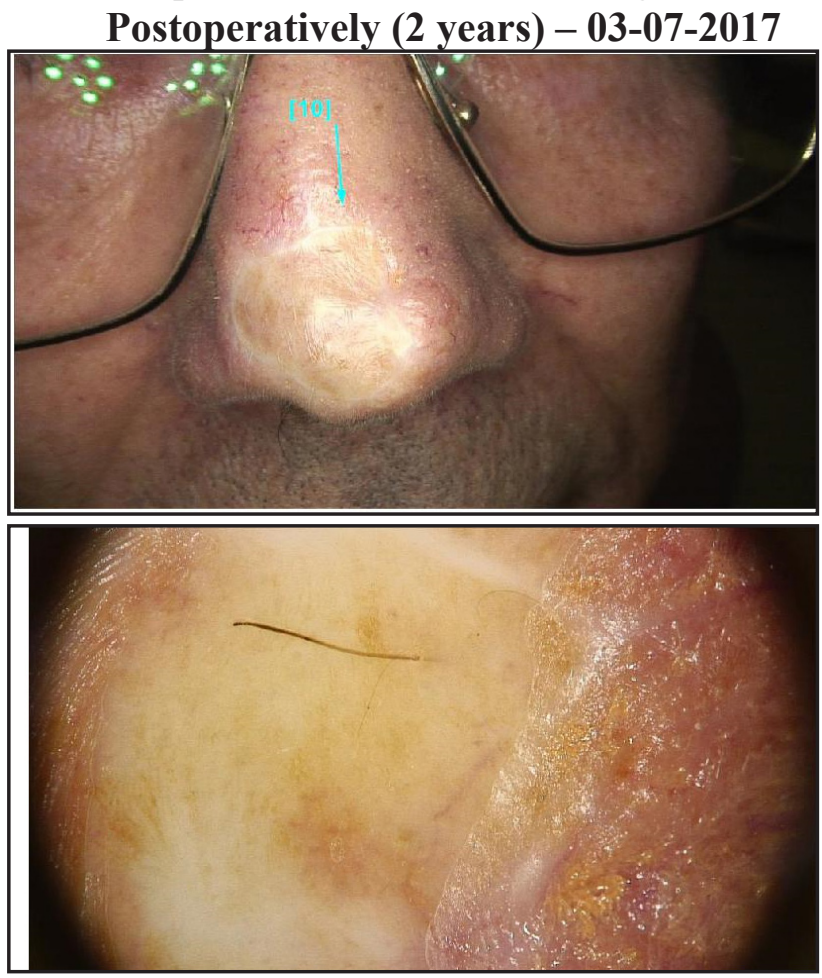

Clinical appearance

Dermatoscopic appearance

Status after surgery.No elements of local recurrence.

CASE II.

Before surgery-04-08-2016
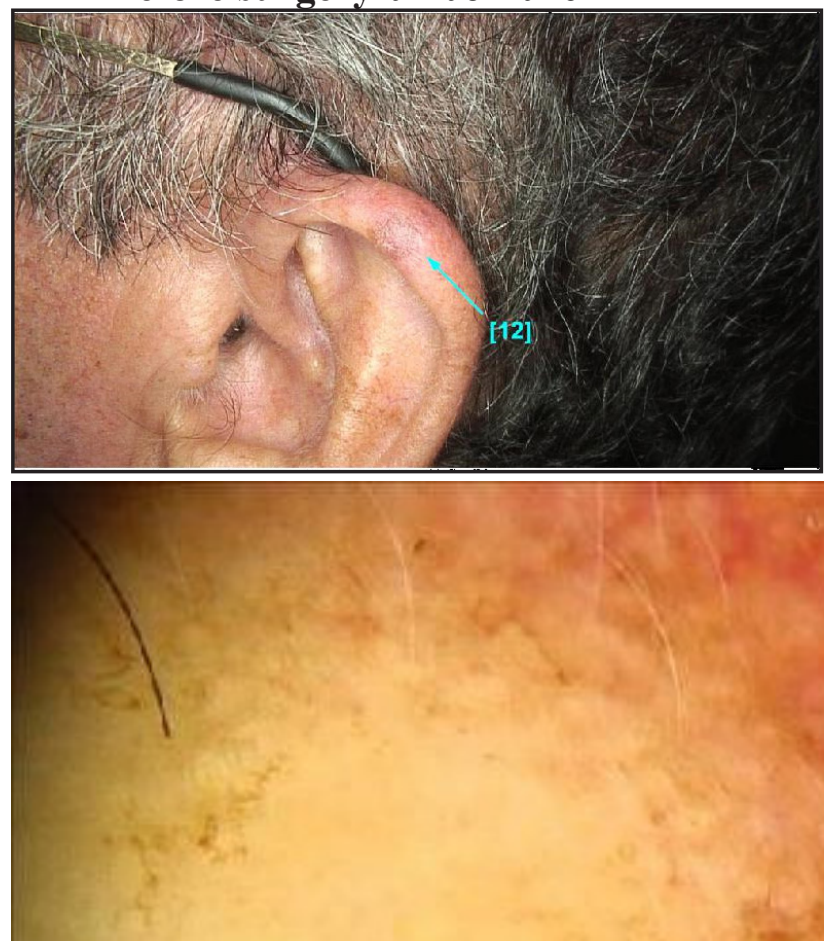

Clinical appearance

Dermatoscopic appearance 
Dermatoscopic diagnosis: In observation pigmentary actinic keratosis (rosette sign + diffuse pigmentation). maligna;

Differential diagnosis with: Lentigo

Recommendations: For preventive considerations, surgical excision with side edges 3-4 $\mathrm{mm}$ from the apparent margin and in depth to the cartilaginous plane, with the direct closure of the post-excisional defect.

The result of the histopathological examination was in conformity with the digital dermatoscopy exam.

\section{Postoperatively (1 year) -12-10-2017}

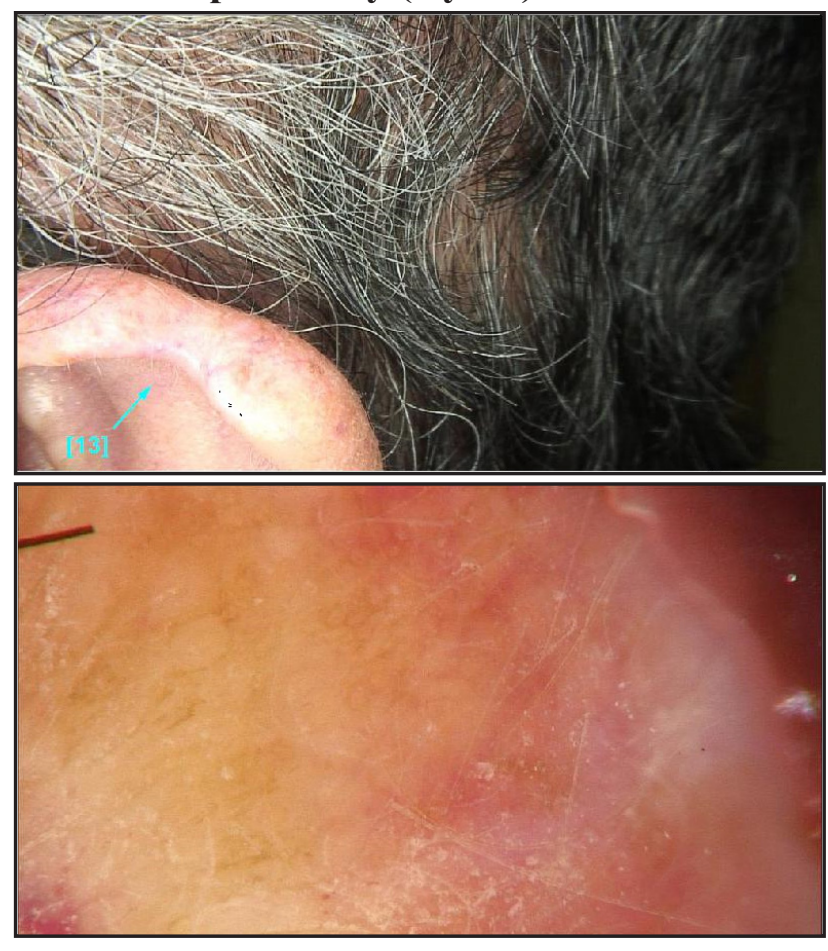

Clinical appearance Dermatoscopic appearance

Status after surgery.No elements of local recurrence.

\section{Dscussion}

The data obtained in this study come to confirm the value of digital dermatoscopy in the diagnosis, treatment and postoperative follow-up of precancerous skin lesions .

From the literature that we have studied, results conclusions and percentages that practically overlap with ours, although the experience of the clinic in which the study was conducted is modest in terms of the implementation of digital dermatoscopy.

The value of digital dermatoscopy is particularly useful in preoperative diagnosis, as it removes the "surprises" that can occur after a surgical excision performed only by clinical criteria (the lateral and deep edges) and which can often require re-excitement (completing the excision), thus exposing the patient to undesirable risks.

\section{Enclusions}

Dermatoscopy is a valuable non-invasive technique both in the diagnosis, treatment, and in the proper tracking of precancerous skin lesions.

The value of digital dermatoscopy is underlined by the $97 \%$ compliance of the in vivo diagnosis by dermatoscopy with histopathological diagnosis.

Digital dermatoscopy has become a current examination today and we appreciate that in the future it should become mandatory (according to a diagnosis / treatment protocol) in the treatment of pre-cancerous lesions.

In addition to surgical treatment, we consider that non-surgical treatments (cryotherapy, radiotherapy, laser therapy, photodynamic therapy, topical drugs, chemotherapy) in precancerous tumors of the skin due to the in vivo histopathological diagnosis by digital dermatoscopy will be required in the future.

\section{Acknowledgments:}

Bordeianu I. - for guidance and experience sharing.

Poalelungi T. - for cases and results of dermatoscopy. 
7. Naimer SA, Urkin J. Enhancement of Pediatric Ophthalmologic Diagnosis With a Handheld Polarized Dermatoscope. Clinical pediatrics. 2014;53(6):579-84.

1. Micali G, Lacarrubba F, Massimino D, Schwartz RA. Dermatoscopy: alternative uses in daily clinical practice. J Am Acad Dermatol. 2011;64(6):1135-46.

2. Argenziano G, Soyer HP. Dermoscopy of pigmented skin lesions--a valuable tool for early diagnosis of melanoma. Lancet Oncol. 2001;2(7):443-9.

3. Wolfe JM, Butcher SJ, Lee C, Hyle M. Changing your mind: on the contributions of top-down and bottom-up guidance in visual search for feature singletons. J Exp Psychol Hum Percept Perform. 2003;29(2):483-502.

4. Braun RP, Kaya G, Masouye I, Krischer J, Saurat JH. Histopathologic correlation in dermoscopy: a micropunch technique. Archives of dermatology. 2003;139(3):34951.

5. Ferrara G, Argenyi Z, Argenziano G, Cerio R, Cerroni L, Di Blasi A, et al. The Influence of Clinical Information in the Histopathologic Diagnosis of Melanocytic Skin Neoplasms. PLOS ONE. 2009;4(4):e5375.

6. Soyer HP, Massone C, Ferrara G, Argenziano G. Limitations of histopathologic analysis in the recognition of melanoma: a plea for a combined diagnostic approach of histopathologic and dermoscopic evaluation. Archives of dermatology. 2005;141(2):20911.

8. Stolz W, Braun-Falco O, Bilek P, Landthaler M, Burgdorf W, Cognetta A. Color alas of drmatoscopy. Berlin: Blackwell Wissenschafts-Verlag; 2002.

9. Saphier J. Die Dermatoskopie. III. Mitteilung. Arch Dermatol Syphiol 2002;

10. Goldman L. A simple portable skin microscope for surface microscopy.Arch Dermatol 1958;78:246-247.

11. MacKie R. An aid to the preoperative assessment of pigmented lesions of the skin. Br J Dermatol 1971;85:232-238.

12. Bahmer F, Fritsch P, Kreusch J, Pehamberger $\mathrm{H}$, et al. Ter-minology in surface microscopy. Consensus Meeting of the Committee on Analytical Morphology of the Arbeitsgemeinschaft Dermatologische Forschung, Hamburg, Federal Republic of Germany, Nov 17, 1989. JAm Acad Dermatol 1990;23:11591162 .

13. Argenziano G, Soyer HP, Chimenti S, et al. Dermoscopy of pigmented skin lesions: results of a consensus meeting via the Internet. J Am Acad Dermatol 2003;48:679693. 